\title{
Switch therapy in hospitalized patients with community-acquired pneumonia: Tigecycline vs. Levofloxacin
}

\author{
Julio A Ramirez ${ }^{1,3 *}$, Angel C Cooper², Timothy Wiemken ${ }^{1}$, David Gardiner ${ }^{2}$ and Timothy Babinchak ${ }^{2}$ \\ For the 308 Study Group
}

\begin{abstract}
Background: Switch therapy is a management approach combining early discontinuation of intravenous (IV) antibiotics, switch to oral antibiotics, and early hospital discharge. This analysis compares switch therapy using tigecycline versus levofloxacin in hospitalized patients with community-acquired pneumonia (CAP).

Methods: A prospective, randomized, double-blind, Phase 3 clinical trial; patients were randomized to IV tigecycline (100 mg, then $50 \mathrm{mg} \mathrm{q12h)} \mathrm{or} \mathrm{IV} \mathrm{levofloxacin} \mathrm{(500} \mathrm{mg} \mathrm{q24h).} \mathrm{Objective} \mathrm{criteria} \mathrm{were} \mathrm{used} \mathrm{to} \mathrm{define} \mathrm{time} \mathrm{to} \mathrm{switch}$ therapy; patients were switched to oral levofloxacin after $\geq 6$ IV doses if criteria met. Switch therapy outcomes were assessed within the clinically evaluable (CE) population.

Results: In the CE population, 138 patients were treated with IV tigecycline and 156 were treated with IV levofloxacin. The proportion of the population that met switch therapy criteria was 67.4\% (93/138) for tigecycline and $66.7 \%(104 / 156)$ for levofloxacin. The proportion that actually switched to oral therapy was $89.9 \%(124 / 138)$ for tigecycline and $87.8 \%(137 / 156)$ for levofloxacin. Median time to actual switch therapy was 5.0 days each for tigecycline and levofloxacin. Clinical cure rates for patients who switched were $96.8 \%$ for tigecycline and $95.6 \%$ for levofloxacin. Corresponding cure rates for those that met switch criteria were $95.7 \%$ for tigecycline and $92.3 \%$ for levofloxacin.
\end{abstract}

Conclusions: Switch therapy outcomes in hospitalized patients with CAP receiving initial IV therapy with tigecycline are comparable to those of patients receiving initial IV therapy with levofloxacin. These data support the use of IV tigecycline in hospitalized patients with CAP when the switch therapy approach is considered.

ClinicalTrials.gov Identifier: NCT00081575

Keywords: Tigecycline, Levofloxacin, CAP, Oral switch

\section{Background}

Community-acquired pneumonia (CAP), one of the most common infectious diseases managed by clinicians, is a key source of morbidity, mortality and expenditure of health-care resources [1,2]. Empiric antimicrobial recommendations, aimed at use of "correct-spectrum" coverage, are outlined in guidelines adopted by numerous global agencies [3-10], including evidence-based guidelines

\footnotetext{
* Correspondence: j.ramirez@louisville.edu

'University of Louisville, Louisville, KY, USA

${ }^{3}$ Division of Infectious Diseases, University of Louisville, 501 E Broadway Suite 308, Louisville, KY 40202, USA

Full list of author information is available at the end of the article
}

from the Infectious Diseases Society of America and the American Thoracic Society (IDSA/ATS) [11]. Yet, a recent compliance evaluation conducted in 36 hospitals in 14 countries found that practitioners do not routinely follow the guidelines; improvement is needed, especially in the areas of CAP prevention, initial empirical therapy, and switch from intravenous (IV) to oral antibiotics [12].

Identifying patients who may be candidates for early switch from IV to oral antibiotic therapy and potentially early hospital discharge is important in the management of CAP [12-14]. Switch therapy is generally considered appropriate and safe when four criteria indicative of clinical stability are met, including improvement in cough 
and respiratory distress-related symptoms, defervescence of fever for at least 8 hours, normalizing white blood cell count, and adequate oral intake and gastrointestinal tract absorption. The majority of patients with non-severe disease can switch from IV to oral therapy within 2-4 days. Previous prospective studies of hospitalized CAP patients have shown that clinical cure rates with switch therapy were high [15-17], even in patients whose pneumonia is complicated by bacteremia [18]. Switch therapy also has health economic benefits via reduction in costs due to drug administration and decreased length of hospital stay [13].

Tigecycline is a first-in-class expanded broad-spectrum, glycylcycline antibiotic [19], and is approved by the U.S. FDA for the treatment of community-acquired bacterial pneumonia caused by penicillin-susceptible Streptococcus pneumoniae, beta-lactamase negative Haemophilus influenzae, and Legionella pneumophila. Tigecycline is well distributed into lung tissues and fluids [20], and has been shown to be as effective as levofloxacin in two global Phase 3 clinical trials of CAP [21].

In the current analysis, data from one of the pivotal prospective trials that compared the efficacy of tigecycline vs. levofloxacin in hospitalized patients with CAP [22] was used to assess outcomes in patients switched to oral therapy. The primary objectives of this study were 1) to compare clinical cure rates for hospitalized patients with CAP treated empirically with IV tigecycline followed by a switch to oral levofloxacin versus those treated empirically with IV levofloxacin followed by a switch to oral levofloxacin and 2) to compare time to switch therapy for hospitalized patients with CAP treated empirically with IV tigecycline versus those treated empirically with IV levofloxacin.

\section{Methods}

\section{Study design and treatment}

A detailed description of the patients and methodology for tigecycline study 308 are described elsewhere [22]. In brief, a Phase 3, multicenter, randomized, double-blind (third party unblinded) clinical trial was conducted to compare the efficacy and safety of tigecycline with levofloxacin in hospitalized adults with CAP. The current analysis focused on comparing efficacy outcomes for two subgroups of patients: those receiving oral switch therapy and those not switched to an oral antibiotic.

Patients were randomly assigned $(1: 1)$ to initially receive either IV tigecycline (100 mg initially followed by $50 \mathrm{mg}$ every $12 \mathrm{~h}$ ) or IV levofloxacin (500 mg every $24 \mathrm{~h}$ ) [22]. A reduced dose of levofloxacin was given to patients with impaired renal function. After at least 3 days of IV therapy (6 IV doses), patients in either treatment group could be switched to oral levofloxacin treatment (500 mg every $24 \mathrm{~h}$ ), at the investigator's discretion, after meeting specified criteria (see below). The total duration of study therapy was 7 to 14 days at the investigator's discretion.

\section{Patient eligibility}

Hospitalized adult patients (at least 18 years of age) of either gender with CAP who initially required IV antibiotics could participate. Each patient had a documented fever within $24 \mathrm{~h}$ of enrolment, a new lung infiltrate confirmed by chest radiograph within $48 \mathrm{~h}$ of receiving the first dose of study medication and at least 2 common signs and/or symptoms suggestive of CAP (e.g., cough, production of purulent sputum, WBC $\left.>10 \times 10^{9} / \mathrm{L}\right)$. Patients were not allowed to participate in the study for any of the following reasons: recent hospitalization within 14 days, residence in a long-term care facility for $\geq 14$ days before onset of symptoms, required treatment in the ICU at the time of randomization, and those with known or suspected Pseudomonas aeruginosa infection.

\section{Oral switch criteria}

In study 308, the specific criteria listed below were to be satisfied at the time the patient was switched to oral levofloxacin. The improvements in signs and symptoms of pneumonia (e.g., cough, shortness of breath, temperature) were to be interpreted by the investigator in relation to the prior day, not baseline. The specific criteria, as judged by the investigator, indicating that clinical stability had been achieved and that the patient was a candidate for oral therapy included the following:

1. Cough and shortness of breath improving.

2. Patient afebrile for $\geq 24$ hours (oral temperature $<37.8^{\circ} \mathrm{C} / 100^{\circ} \mathrm{F}$, or equivalent for axillary, tympanic, or rectal/core temperature), on 2 or more measurements at least 24 hours apart, with no known spiking of temperature during that interval.

3. White blood cell count improving (no change was required if the $\mathrm{WBC}$ count was within normal range): $>10 \%$ decrease if initial WBC count was elevated $\left(>10 \times 10^{9} / \mathrm{L}\right) ;>5 \%$ decrease in immature neutrophils (bands) if initially $>15 \%$; or $>10 \%$ increase if patient was initially leukopenic (WBC $\left.<4.5 \times 10^{9} / \mathrm{L}\right)$.

4. Oral intake and gastrointestinal tract absorption adequate.

Oral intake was defined as a patient taking food by mouth without gastrointestinal intolerance on any day. For the other three objective criteria of WBC count, cough/dyspnea, and temperature, the patient was considered to reach criteria based on change from baseline or the prior day. In this analysis, patients had to meet all three objective criteria and oral intake criteria to be 
considered a candidate for oral therapy. For WBC, patients could meet any of the criteria below, up to the last day of therapy visit:

- Previous WBC is normal and first WBC value post-baseline is normal

- Previous WBC is above normal and WBC decreases by $10 \%$ (second value)

- Previous WBC is below normal and WBC increases by $10 \%$ (second value)

- If previous bands $>15 \%$ and bands decrease by $5 \%$ (second value)

For cough and dyspnea, patients could meet any of the criteria below from the first visit up to last day of therapy visit:

- Cough and dyspnea are absent

- Cough and dyspnea improve from the previous visit (second visit)

- Cough and dyspnea are unchanged from previous visit and improved from baseline

For temperature, patient met criteria if temperature was $<37.8{ }^{\circ} \mathrm{C}$ with a previous temperature $<37.8{ }^{\circ} \mathrm{C}$ (second visit). If multiple temperatures were taken during same relative day, the maximum temperature was used.

\section{Time to clinical stability}

Patients were considered to have clinical improvement on the day they met all 4 criteria for switch therapy according to the study protocol. Patients who satisfied the criteria for switch therapy during the first 3 days of hospital treatment were considered to have early clinical stability. Patients who met the criteria for switch therapy from days 4 to 7 of hospital treatment were considered to have late clinical stability.

\section{Analysis population and efficacy assessments}

The present analysis is restricted to patients categorized as clinically evaluable (CE). Patients who had clinical evidence of CAP by meeting the minimal disease criteria were considered to be CE if they satisfied inclusion and exclusion criteria, received no more than one dose of a non-once, daily, non-study antibacterial agent (single agent or combination therapy) to treat the current episode of CAP before the first dose of study drug, did not receive other concomitant systemic antimicrobial therapy unless a treatment failure, received at least 2 full days of study drug if clinical failure or 5 full days of study drug (IV plus oral) if clinical cure, were adherent with therapy (i.e., $\geq 80 \%$, but $\leq 120 \%$ of medication administered), had an assessment of cure or failure (and not indeterminate) at the test-of-cure (TOC) visit, and the study blind was maintained. The primary efficacy endpoint was clinical response in the $\mathrm{CE}$ population at the TOC visit.

\section{Statistical analysis}

Categorical baseline demographic and medical variables were analyzed using Fisher's exact test and chi-square test. Continuous variables were compared using a oneway analysis of variance (ANOVA) model with treatment as a factor. The time to meet oral switch criteria was analyzed by the Kaplan-Meier approach using log-rank test for differences in survival curves.

Overall clinical response rates were compared for hospitalized patients with CAP treated empirically with IV tigecycline followed by a switch to oral levofloxacin versus those treated empirically with IV levofloxacin followed by a switch to oral levofloxacin, using Fisher's exact test. Clinical response was also evaluated for each treatment for patients in the following groups: $I V / P O$ Switch (all patients who switched to PO therapy) versus IV-only (patients not switched to PO therapy) and Met Switch Criteria (regardless of whether the patient was switched to oral therapy) versus Did Not Meet Switch Criteria. $P$ values $<0.05$ were considered significant. Statistical analysis was performed by the Global Biostatistics and Programming department of Wyeth Research, Collegeville, PA, which was acquired by Pfizer Inc in October of 2009.

The study protocol was reviewed and approved by each investigator's independent ethics committee or institutional review board in accordance with local regulations and good clinical practices. Written informed consent was obtained from each patient or his or her guardian before initiation of any study procedure according to the guidelines of each institution.

\section{Results}

\section{Patients and treatment}

A total of 425 patients were available for the Intent To Treat population analysis in study 308. A total of 294 patients were included in the $\mathrm{CE}$ population. The $\mathrm{CE}$ population was comprised of 138 hospitalized patients with CAP treated empirically with IV tigecycline and 156 hospitalized patients with CAP treated empirically with IV levofloxacin (Table 1). Nearly two-thirds (67.0\%; [197/ 294]) of patients met objective IV to oral switch criteria per study protocol (93/138, 67.4\% tigecycline and 104/156, $66.7 \%$ levofloxacin; Table 1). Switch to oral therapy was performed in $88.8 \%(261 / 294)$ of patients (124/138, $89.9 \%$ tigecycline; 137/156, 87.8\% levofloxacin; Table 1). Demographic and baseline medical characteristics, grouped by patients who were switched or not switched to oral therapy, are outlined in Table 2.

The median time to clinical stability (i.e., time when all criteria for oral switch were met) for the CE population 
Table 1 Clinically Evaluable Population

\begin{tabular}{|c|c|c|c|}
\hline Number of patients, $\mathbf{n}(\%)$ & Tigecycline $(\mathrm{N}=138)$ & Levofloxacin $(\mathrm{N}=156)$ & Total $(\mathrm{N}=294)$ \\
\hline Patients switched to oral therapy & $124(89.9 \%)$ & $137(87.8 \%)$ & $261(88.8 \%)$ \\
\hline Patients not switched to oral therapy & $14(10.1 \%)$ & $19(12.2 \%)$ & $33(11.2 \%)$ \\
\hline Patients meeting switch criteria as determined by present analysis & $93(67.4 \%)$ & $104(66.7 \%)$ & $197(67.0 \%)$ \\
\hline Patients not meeting switch criteria as determined by present analysis & $45(32.6 \%)$ & $52(33.3 \%)$ & $97(33.0 \%)$ \\
\hline Patients switched without meeting switch criteria as determined by present analysis & $31(22.5 \%)$ & $33(21.2 \%)$ & $64(21.8 \%)$ \\
\hline
\end{tabular}

was 4 days each for tigecycline and levofloxacin $(P=0.220$; Figure 1$)$. Nearly $50 \%$ of patients in both treatment groups were categorized as having early clinical stability. The median time to actual switch to oral therapy was 5.0 days each for tigecycline- and levofloxacin-treated patients $(P=0.274$; Figure 2$)$. Figure 3 illustrates the number of patients in each group who met IV to oral switch criteria, as determined by the present analysis, by treatment day. Of note, 64 patients who were switched to oral therapy (31 tigecycline, 33 levofloxacin) had not met objective criteria for IV to oral switch, as determined by the present analysis. Thirteen patients who met objective criteria for IV to oral switch were not switched to oral therapy (4 tigecycline, 9 levofloxacin). Although the study protocol had easy to follow and welldefined switch therapy criteria, in 77 of the 294 patients evaluated (26\%), the switch therapy criteria were not followed by the investigators.

\section{Clinical efficacy}

For patients switched to oral therapy by the investigator, overall cure rates in the CE population were similar between tigecycline and levofloxacin (tigecycline 96.8\% [120/124]; levofloxacin 95.6\% [131/137]) $(P=0.752$; Figure 4). In patients not switched, cure rates were low, as expected, in both treatment groups (tigecycline 35.7\% [5/ 14]; levofloxacin 26.3\% [5/19]) $(P=0.707)$. Among patients who met switch criteria as determined by the study protocol, the clinical cure rate was $95.7 \%$ (89/93) for tigecycline and $92.3 \%(96 / 104)$ for levofloxacin $(P=0.382$; Figure 4). Corresponding rates for patients who did not meet switch criteria were $80.0 \%(36 / 45)$ and $76.9 \%(40 /$ $52)$, respectively $(P=0.807)$.

\section{Discussion}

This study indicates no significant difference in the clinical cure rates for hospitalized patients with CAP treated

Table 2 Baseline Demographic and Medical Characteristics for CE Population: Switched vs. Not Switched from IV to Oral Therapy

\begin{tabular}{|c|c|c|c|c|c|c|}
\hline \multirow{2}{*}{ Characteristic } & \multicolumn{3}{|c|}{ Switched (IV to oral) } & \multicolumn{3}{|c|}{ No Switch (IV only) } \\
\hline & Tigecycline $\mathrm{N}=124$ & Levofloxacin N= 137 & $P$-value & Tigecycline $\mathrm{N}=14$ & Levofloxacin $\mathrm{N}=19$ & $P$-value \\
\hline Age, yrs, mean $\pm S D$ & $55.2 \pm 16.4$ & $54.0 \pm 20.4$ & 0.613 & $60.1 \pm 18.1$ & $63.1 \pm 17.2$ & 0.634 \\
\hline Male, n (\%) & $64(51.6)$ & $83(60.6)$ & 0.145 & $9(64.3)$ & $13(68.4)$ & 0.803 \\
\hline Ethnic origin, n (\%) & & & 0.189 & & & 0.620 \\
\hline White & $80(64.5)$ & $87(63.5)$ & & $9(64.3)$ & $9(47.4)$ & \\
\hline Hispanic & $29(23.4)$ & $34(24.8)$ & & $5(35.7)$ & $7(36.8)$ & \\
\hline Black & $14(11.3)$ & $9(6.6)$ & & 0 & $1(5.3)$ & \\
\hline Asian & 0 & $4(2.9)$ & & 0 & $1(5.3)$ & \\
\hline Other & $1(0.8)$ & $3(2.2)$ & & 0 & $1(5.3)$ & \\
\hline Fine Score, n (\%) & & & 0.452 & & & 0.816 \\
\hline 1 & $28(22.6)$ & $41(29.9)$ & & $3(21.4)$ & $2(10.5)$ & \\
\hline$\|$ & $43(34.7)$ & $42(30.7)$ & & $1(7.1)$ & $1(5.3)$ & \\
\hline III & $33(26.6)$ & $29(21.2)$ & & $5(35.7)$ & $7(36.8)$ & \\
\hline IV & $20(16.1)$ & $25(18.2)$ & & $5(35.7)$ & $9(47.4)$ & \\
\hline Prior antibiotic failure, $\mathrm{n}(\%)$ & $2(1.6)$ & $4(3.0)$ & 0.471 & 0 & $1(5.3)$ & 0.383 \\
\hline Therapy duration, days, mean \pm SD & $11.39 \pm 2.0$ & $11.26 \pm 2.0$ & 0.599 & $6.7 \pm 3.1$ & $6.3 \pm 3.7$ & 0.746 \\
\hline Presence of multilobar infiltrates, $n$ (\%) & $32(25.8)$ & $22(16.1)$ & - & $7(50.0)$ & $7(36.8)$ & - \\
\hline Presence of altered mental status, $\mathrm{n}(\%)$ & $2(1.6)$ & $5(3.6)$ & - & 0 & $1(5.3)$ & - \\
\hline
\end{tabular}




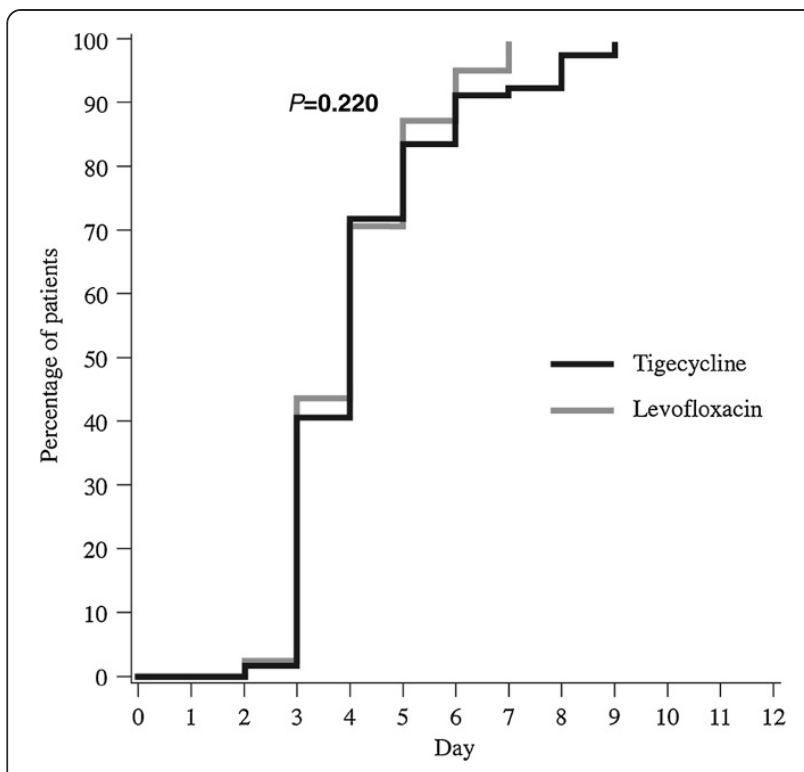

Figure 1 Days until patients met objective criteria for IV to oral switch.

empirically with IV tigecycline followed by a switch to oral levofloxacin versus those treated empirically with IV levofloxacin followed by a switch to oral levofloxacin. There was also no significant difference in the time to switch therapy for hospitalized patients with CAP treated empirically with IV tigecycline versus those treated empirically with IV levofloxacin. The median time to meet criteria for switch therapy was 4 days for both study groups. Our study findings indicating equal clinical activity and equal time to switch therapy for each study arm

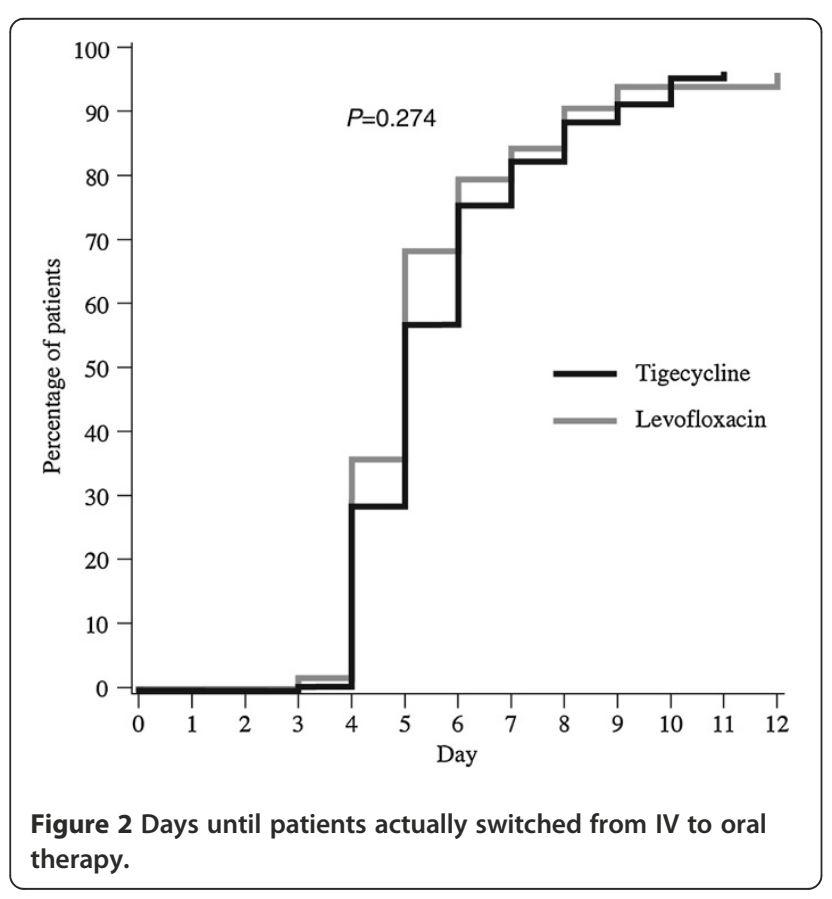

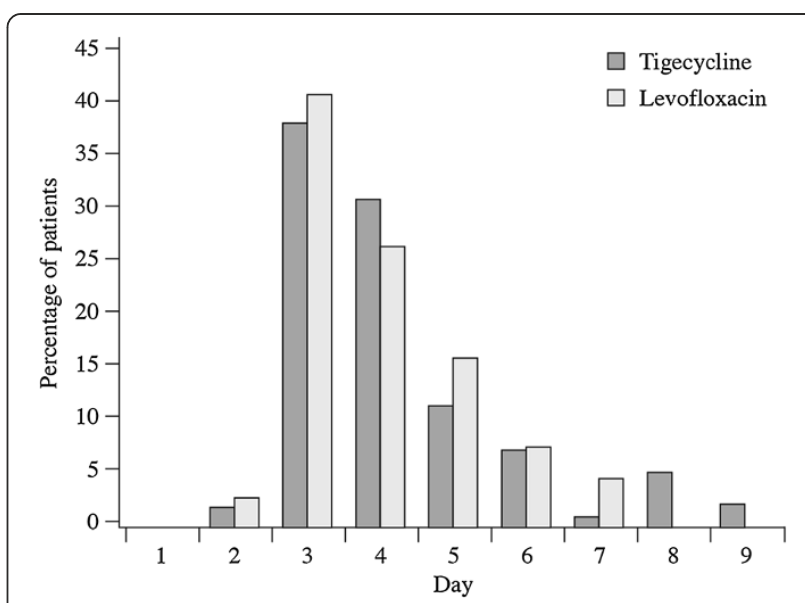

Figure 3 Number of patients meeting objective IV to oral switch criteria.

suggest that both antibiotics have similar activity against pulmonary pathogens.

Nearly half of all hospitalized patients with CAP met criteria for switch therapy by day 3 of hospitalization. These data indicating early clinical stability in a significant number of hospitalized patients with CAP, are similar to those reported in prior studies on CAP [15-18]. It is important to recognize these groups of patients with early clinical improvement since they can be targeted for an approach for early switch therapy and early hospital discharge. National guidelines indicate that patients can be safely switched to oral therapy on the same day they meet criteria for clinical stability. In our study, the median time for patients to meet criteria for clinical stability was 4 days in both study arms, but the time to actual switch was 5 days in both study arms. This one-day delay

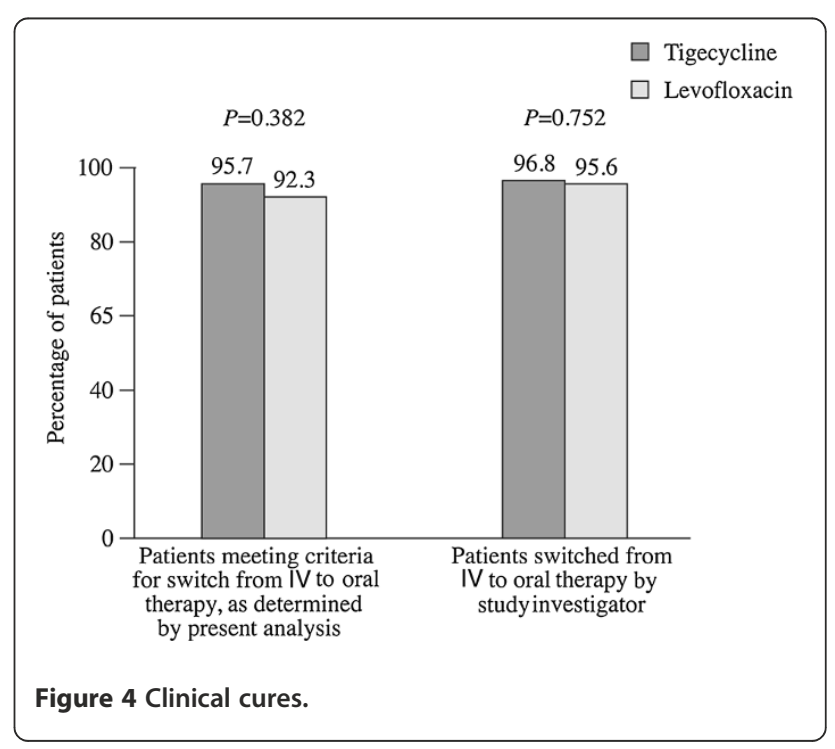


to implement oral switch will not offer any clinical benefit and is likely to delay hospital discharge. A delayed switch to oral therapy may also be associated with a number of potential detrimental effects to the patient, the caregiver, and the healthcare system. Although our analysis did not capture these parameters, delayed hospital discharge may increase the patient's risk for nosocomial infections [16].

Our data indicate that patients who were less severely ill (e.g., Fine Score I-III) were more likely to reach criteria for switch therapy. These findings are in agreement with a recent evaluation of severity of disease at time of hospitalization and time to clinical stability for patients with CAP [23]. The authors found that as severity of disease increased, measured by CRB-65 and Fine Score, time to clinical stability also increased.

This study has several limitations. Although the protocol defined objective criteria for switch therapy, the actual switch to oral therapy was at the discretion of the investigator. The fact that, in $26 \%$ of the patients the investigators failed to follow the switch therapy criteria as defined in the protocol, has the potential to induce selection bias. As explained in the Methods section, we used only the clinically evaluable population for our analysis. Since we did not use the Intent To Treat population, this may have incorporated some bias into the results. The study did not collect long-term efficacy data beyond the test of cure visit at 7-23 days after the last day of antibiotic therapy. Because of the short follow-up in some patients there is a possibility for missing cases with relapse of infection after switch. In this study, we evaluated the role of two antibiotics in time to clinical stability. Confounding factors for this association include the bacterial etiology of CAP as well as host factors. The generalizability of our data is limited due to the fact that patients admitted to the ICU or patients with risk factors for Pseudomonas aeruginosa were excluded from the trial. One strength of this study is that randomization into the two study arms may limit confounding effects.

Because duration of hospitalization is an important driver in the costs of CAP, implementing an oral switch protocol will provide economic benefits. Although not all patients who are candidates for oral switch therapy can be discharged early, adoption of universal switch therapy in the U.S. is estimated to reduce hospital days by more than a half a million days annually with an overall savings in excess of $\$ 400$ million [13]. Adoption of an oral switch strategy is also an important component of antibiotic stewardship programs, which are aimed, in part, at minimizing emergence of multiple-drug resistant bacteria [24-26]. Despite data that support the efficacy of oral switch therapy [15-18, 27-30], practitioners often fail to assess if a patient is an appropriate candidate, or elect to continue IV therapy because the patient is improving.

\section{Conclusion}

In conclusion, the tigecycline approach to switch therapy is equivalent to a levofloxacin approach to switch therapy. This study supports the use of short-course tigecycline in hospitalized patients with community-acquired pneumonia followed with an early switch to oral therapy in appropriate patients.

\section{Competing interests}

AC is an employee of Pfizer Inc. DG and TB are former employees of Pfizer, Inc. JAR is on the Speakers' Bureau for Pfizer Inc. TW has no conflicts to report.

\section{Author contributions}

JAR: Contributed to protocol development, data analysis, manuscript development and critical review. ACC: Contributed to protocol development, data analysis, manuscript development and critical review. TW: Contributed to data analysis, manuscript development and critical review. DG: Contributed to protocol development, data analysis, manuscript development and critical review. TB: Contributed to protocol development, data analysis, manuscript development and critical review. All authors read and approved the final manuscript.

\section{Acknowledgments}

The original study and the present analysis were sponsored and supported by Wyeth Research, Collegeville, PA, USA, which was acquired by Pfizer Inc in October 2009. The authors thank Dr. Gary Dukart, formerly of Pfizer Inc., for his invaluable contributions to the present study and analysis, as well as to the preparation of this manuscript. Editorial support for the preparation of this manuscript, funded by Pfizer Inc., was provided by Annie Jones of Upside Endeavors, LLC (Sanatoga, PA) and Patricia Fonseca of UBC Scientific Solutions (Horsham, UK).

\section{Author details}

'University of Louisville, Louisville, KY, USA. ${ }^{2}$ Pfizer Inc, Collegeville, PA, USA. ${ }^{3}$ Division of Infectious Diseases, University of Louisville, 501 E Broadway Suite 308, Louisville, KY 40202, USA.

Received: 6 October 2011 Accepted: 8 March 2012

Published: 19 July 2012

\section{References}

1. Carbonara S, Monno L, Longo B, Angarano G: Community-acquired pneumonia. Curr Opin Pulm Med 2009, 15(3):261-273.

2. Niederman MS: Community-acquired pneumonia: the U.S. perspective. Semin Respir Crit Care Med 2009, 30(2):179-188

3. Mandell LA, Marrie TJ, Grossman RF, Chow AW, Hyland RH: Summary of Canadian Guidelines for the Initial Management of Community-acquired Pneumonia: An evidence-based update by the Canadian Infectious Disease Society and the Canadian Thoracic Society. Can J Infect Dis. 2000, 11(5):237-248

4. Miyashita N, Matsushima T, Oka M, Japanese Respiratory Society: The JRS guidelines for the management of community-acquired pneumonia in adults: an update and new recommendations. Intern Med 2006, 45(7):419-428

5. Niederman MS: Review of treatment guidelines for community-acquired pneumonia. Am J Med 2004, 117(Suppl 3A):515-57S.

6. Woodhead M: Community-acquired pneumonia guidelines-an international comparison: a view from Europe. Chest 1998, 113(3 Suppl):183S-187S.

7. Woodhead M, Macfarlane J: Local antibiotic guidelines for adult community-acquired pneumonia (CAP): a survey of UK hospital practice in 1999. J Antimicrob Chemother 2000, 46(1):141-143.

8. British Thoracic Society Standards of Care Committee (2001): BTS Guidelines for the Management of Community Acquired Pneumonia in Adults. Thorax 2001, 56(Suppl 4):IV1-64

9. Memish ZA, Shibl AM, Ahmed QA: Guidelines for the management of community-acquired pneumonia in Saudi Arabia: a model for the Middle East region. Int J Antimicrob Agents 2002, 20(Suppl 1):S1-S12. 
10. Gialdroni Grassi G, Bianchi L: Guidelines for the management of community-acquired pneumonia in adults. Italian Society of Pneumology. Italian Society of Respiratory Medicine. Italian Society of Chemotherapy. Monaldi Arch Chest Dis 1995, 50(1):21-27.

11. Mandell LA, Wunderink RG, Anzueto A, Bartlett JG, Campbell GD, Dean NC, et al: Infectious Diseases Society of America/American Thoracic Society consensus guidelines on the management of community-acquired pneumonia in adults. Clin Infect Dis 2007, 44(Suppl 2):S27-S72.

12. Ramirez JA: Worldwide perspective of the quality of care provided to hospitalized patients with community-acquired pneumonia: results from the CAPO international cohort study. Semin Respir Crit Care Med 2005, 26 (6):543-552.

13. Ramirez JA: Managing antiinfective therapy of community-acquired pneumonia in the hospital setting: focus on switch therapy. Pharmacotherapy 2001, 21(7 Pt 2):79S-82S

14. Peyrani P, Christensen D, LaJoie AS, Nakamatsu R, Arnold F, Schulz P, et al: Antibiotic therapy of hospitalized patients with community-acquired pneumonia: an international perspective from the CAPO Cohort Study. $J$ Ky Med Assoc 2006, 104(11):513-517.

15. Ramirez JA, Vargas S, Ritter GW, Brier ME, Wright A, Smith S, et al: Early switch from intravenous to oral antibiotics and early hospital discharge: a prospective observational study of 200 consecutive patients with community-acquired pneumonia. Arch Intern Med 1999, 159(20):2449-2454.

16. Ramirez JA: Switch therapy in community-acquired pneumonia. Diagn Microbiol Infect Dis 1995, 22(1-2):219-223.

17. Ramirez JA, Srinath L, Ahkee S, Huang A, Raff MJ: Early switch from intravenous to oral cephalosporins in the treatment of hospitalized patients with community-acquired pneumonia. Arch Intern Med 1995, 155 (12):1273-1276.

18. Ramirez JA, Bordon J: Early switch from intravenous to oral antibiotics in hospitalized patients with bacteremic community-acquired Streptococcus pneumoniae pneumonia. Arch Intern Med 2001, 161(6):848-850.

19. McKeage K, Keating GM: Tigecycline: in community-acquired pneumonia. Drugs 2008, 68(18):2633-2644.

20. Conte JEJ, Golden JA, Kelly MG, Zurlinden E: Steady-state serum and intrapulmonary pharmacokinetics and pharmacodynamics of tigecycline. Int J Antimicrob Agents 2005, 25(6):523-529.

21. Tanaseanu C, Bergallo C, Teglia O, Jasovich A, Oliva ME, Dukart G, et al: Integrated results of 2 phase 3 studies comparing tigecycline and levofloxacin in community-acquired pneumonia. Diagn Microbiol Infect Dis 2008, 61(3):329-338.

22. Bergallo $C$, Jasovich A, Teglia O, Oliva ME, Lentnek A, de Wouters $L$, et al: Safety and efficacy of intravenous tigecycline in treatment of community-acquired pneumonia: results from a double-blind randomized phase 3 comparison study with levofloxacin. Diagn Microbiol Infect Dis 2009, 63(1):52-61.

23. Arnold FW, Brock GN, Peyrani P, Rodriguez EL, Diaz AA, Rossi P, Ramirez JA: Predictive accuracy of the pneumonia severity index vs CRB-65 for time to clinical stability: Results from the Community-Acquired Pneumonia Organization (CAPO) International Cohort Study. Respir Med 2010, 104 (11):1736-1743.

24. Antibiotic stewardship programs curb resistance. Healthcare Benchmarks Qual Improv 2008, 15(11):114-5.

25. Dos Santos RP, Magedanz L, Siliprandi EM: Antimicrobial stewardship programs must apply to all. Infect Control Hosp Epidemiol 2009, 30(2):205-207.

26. Drew RH: Antimicrobial stewardship programs: how to start and steer a successful program. J Manag Care Pharm 2009, 15(2 Suppl):S18-S23.

27. Mertz D, Koller M, Haller P, Lampert ML, Plagge H, Hug B, et al: Outcomes of early switching from intravenous to oral antibiotics on medical wards. J Antimicrob Chemother 2009, 64(1):188-199.

28. Barth J, Stauch K, Landen H: Efficacy and tolerability of sequential intravenous/oral moxifloxacin therapy in pneumonia: results of the first post-marketing surveillance study with intravenous moxifloxacin in hospital practice. Clin Drug Investig 2005, 25(11):691-700.

29. Pelly L: IV-to-oral switch therapy for community-acquired pneumonia requiring hospitalization: focus on gatifloxacin. Adv Ther 2002. 19(5):229-242
30. Furlanut M, Brollo L, Lugatti E, Di Qual E, Dolcet F, Talmassons G, et al: Pharmacokinetic aspects of levofloxacin $500 \mathrm{mg}$ once daily during sequential intravenous/oral therapy in patients with lower respiratory tract infections. J Antimicrob Chemother 2003, 51(1):101-106.

doi:10.1186/1471-2334-12-159

Cite this article as: Ramirez et al:: Switch therapy in hospitalized patients with community-acquired pneumonia: Tigecycline vs. Levofloxacin. $B M C$ Infectious Diseases 2012 12:159.

\section{Submit your next manuscript to BioMed Central and take full advantage of:}

- Convenient online submission

- Thorough peer review

- No space constraints or color figure charges

- Immediate publication on acceptance

- Inclusion in PubMed, CAS, Scopus and Google Scholar

- Research which is freely available for redistribution 